\title{
The Budget Impact of Introducing Tildrakizumab to a United States Health Plan for Managing Moderate-to-Severe Plaque Psoriasis
}

\author{
Justin Carrico ${ }^{1} \cdot$ Yang Zhao $^{2} \cdot$ Xiaoying Jia $^{3} \cdot$ Thor-Henrik Brodtkorb $^{4} \cdot$ Alan Mendelsohn $^{5} \cdot$ Simon Lowry $^{6}$
}

Published online: 26 March 2020

(c) The Author(s) 2020

\begin{abstract}
Objective The aim of this study was to evaluate the budget impact of introducing tildrakizumab for moderate-to-severe plaque psoriasis from a US health plan perspective.

Methods A budget impact model estimated costs before and after the adoption of tildrakizumab to a hypothetical US health plan with 1 million covered lives over 5 years. Additionally, the model included adalimumab, brodalumab, etanercept, guselkumab, ixekizumab, secukinumab, ustekinumab, and apremilast; biosimilars were not included. Model input data were obtained from the published literature, clinical trials, and prescription data. Market uptake for tildrakizumab was assumed as $1 \%$ annually over 5 years. Patients initiating or switching treatments required induction dosing; all others treated required maintenance dosing. The model compared the total annual costs for tildrakizumab versus treatment without tildrakizumab to calculate budget impact in 2018 US dollars. Scenarios exploring alternative assumptions for adverse events and market uptake rates were assessed, and a one-way sensitivity analysis was conducted.

Results Within a health plan of 1 million members with an estimated 1048 patients receiving biologics or apremilast for psoriasis, the total annual health plan cost after introducing tildrakizumab decreased by $\$ 5585, \$ 137,025, \$ 205,538, \$ 274,051$, and $\$ 342,563$ in years $1-5$, respectively, resulting in a cumulative reduction of $\$ 964,763$ over 5 years. The impact on total cost was largely due to drug acquisition costs. The incremental per member per month (PMPM) cost reductions were negligible in year $1, \$ 0.01$ in year $2, \$ 0.02$ in years $3-4$, and $\$ 0.03$ in year 5 . Scenario and sensitivity analyses confirmed the model robustness. Conclusions The introduction of tildrakizumab with a $1 \%$ annual uptake over 5 years has the potential to reduce the cost of treating patients with moderate-to-severe plaque psoriasis for a US health plan.
\end{abstract}

\section{Introduction}

Psoriasis is a chronic immune-mediated disease characterized by skin inflammation, itching, and pain. Patients with psoriasis are also at a higher risk of several comorbid diseases, including cardiovascular diseases, metabolic syndrome, Crohn's disease, and lymphoma [1]. As of 2013, a total of 7.4 million people in the US were affected by psoriasis, with prevalence highest among Caucasians [2]. The estimated annual direct health care costs ranged from $\$ 52$ billion to $\$ 63$ billion in 2013 US dollars, with the majority of

Electronic supplementary material The online version of this article (https://doi.org/10.1007/s41669-020-00208-9) contains supplementary material, which is available to authorized users.

Yang Zhao

yzhao00@hotmail.com

Extended author information available on the last page of the article costs attributed to patients with higher disease severity and with comorbidities [3]. About $90 \%$ of all psoriasis cases are plaque psoriasis [1], 18\% of which are classified as moderate-to-severe, usually defined as having more than $10 \%$ of the body surface area affected by the disease [4, 5].

Many treatment options for moderate-to-severe plaque psoriasis are available, including conventional oral systemic therapies, phototherapy, and, more recently, developed targeted therapies such as biologic treatments and apremilast. Control of psoriasis symptoms is usually measured using the clinician-reported outcome, Psoriasis Area and Severity Index (PASI), which assesses the coverage and severity of plaque psoriasis across zones of the body; PASI 75 (i.e. $75 \%$ improvement in PASI) is commonly used as a measure of treatment response in clinical trials. Biologic treatments and apremilast have been shown to be more effective in improving PASI than conventional systemic therapy or phototherapy [6]. Only one previous study assessed the financial impact of these treatments on payers in the US [7]. However, budget impact analyses are important tools that can be used 


\section{Key Points for Decision Makers}

Although many targeted biologic drugs and other treatments are available for the management of moderateto-severe plaque psoriasis, their impacts on health plan budgets have not been widely available.

Tildrakizumab has been approved for treating moderateto-severe plaque psoriasis, with demonstrated efficacy and safety.

Over a 5-year period, the cumulative impact of adding tildrakizumab to a US health plan was a $\$ 0.02$ per member per month reduction in health plan costs for treating moderate-to-severe plaque psoriasis, compared with a market scenario without tildrakizumab. Biosimilars for the treatments in this analysis are not currently marketed in the US but future uptake of such drugs may impact overall health plan costs.

for assessing the anticipated changes in expenditures when a new treatment is introduced to a health system [8].

Tildrakizumab, a monoclonal antibody that selectively inhibits the p19 subunit of interleukin-23, recently received US FDA approval for the treatment of moderate-to-severe plaque psoriasis. The efficacy of tildrakizumab was demonstrated in two randomized, placebo-controlled, phase III clinical trials, which demonstrated that tildrakizumab was efficacious, versus placebo and etanercept, in achieving improvement in PASI 75 [9]. The affordability of tildrakizumab for US health care payers has not been fully assessed. The objective of this study was to estimate the budget impact of introducing tildrakizumab as a treatment option for moderate-to-severe psoriasis from a US health plan perspective.

\section{Methods}

\subsection{Model Structure}

A budget impact model was developed to estimate the potential impact of introducing tildrakizumab on a US health plan's costs over a period of $1-5$ years. The model started with a hypothetical 1-million-member health plan and then estimated the annual number of moderate-to-severe plaque psoriasis patients treated with biologics or apremilast, as well as the number of patients new to treatment each year. The treated population size remained constant each year for the model estimate. In addition to tildrakizumab, the plaque psoriasis treatments were based on the available treatments at the time of the analysis that had been assessed in previous cost-effectiveness analyses [6] and included adalimumab, apremilast, brodalumab, etanercept, guselkumab, ixekizumab, secukinumab, and ustekinumab. Biosimilars for these treatments are not currently marketed in the US and were thus not included in the analysis. To estimate the budget impact of adding tildrakizumab to the treatment mix, two market scenarios were compared. In scenario 1, patients received current biologic treatments (adalimumab, brodalumab, etanercept, guselkumab, ixekizumab, secukinumab, and ustekinumab) and apremilast based on the current market shares, and in scenario 2 , patients received tildrakizumab (introduced to the formulary over 5 years) and all other approved biologic treatments, as well as apremilast, based on projected annual market shares. Patients received treatments based on dosing per the prescribing information and incurred costs related to treatment acquisition, treatment administration, laboratory testing, and routine clinic visits. The model calculations utilized annual cycles; therefore, patients receiving treatment were assumed to receive the same treatment for the entire year and treatment discontinuation not explicitly modelled.

Costs for treatment acquisition, treatment administration, laboratory and monitoring were calculated in each market scenario for each year of the time horizon and were not discounted. Budget impact was calculated by comparing the total annual health plan costs in scenario 2 with those in scenario 1 . Results were reported in total annual, per member per year (PMPY), per member per month (PMPM), and per treated member per month (PTMPM) costs.

\subsection{Population}

The modelled patient population was the population indicated for tildrakizumab (i.e. adult patients with moderate-to-severe plaque psoriasis who are candidates for systemic therapy or phototherapy) [9]. A 1-million-member initial plan population size was assumed (Table 1). The number of patients eligible for treatment was calculated from the US prevalence estimates for moderate-to-severe plaque psoriasis [1, 2, 4]. Among all patients with moderate-to-severe plaque psoriasis, $20 \%$ were assumed to be treated with biologic treatments or apremilast [10], of whom $36 \%$ were estimated to be new to treatment due to incident psoriasis or switching between treatments [6,7]. All patients treated with tildrakizumab were assumed to be new to treatment in the first year of its introduction (all incurred more frequent dosing during the induction period than in the maintenance period in year 1). In all subsequent years of the model, the portion of tildrakizumab patients new to treatment was consistent with the other modelled treatments (36\%).

The prevalence of moderate-to-severe plaque psoriasis and the proportion of patients treated with biologics was assumed to remain constant. All-cause mortality for the modelled population was not considered. The treated 
population was therefore assumed to remain constant over the modelled time horizon.

\subsection{Costs}

Costs were estimated from a health plan perspective and only considered direct medical and drug acquisition costs. Indirect costs and costs of productivity loss were not considered.

Treated patients incurred drug acquisition costs based on dosing regimens as described in the corresponding prescribing information. The incident (newly diagnosed) psoriasis patients and patients switching biologic treatments incurred drug acquisition costs for the treatment induction period (based on the corresponding phase III clinical trial assessment periods) and costs for doses administered during the maintenance period for the remainder of the year. The remainder of the treated population incurred drug acquisition costs based on the maintenance dosing regimens.

Annual acquisition costs for biologic treatments and apremilast were based on wholesale acquisition costs (WACs) from May 2019 [11] and recommended dosing regimens from the corresponding prescribing information (Table 1). Annual costs were calculated by multiplying the estimated annual treatment doses required by the treatment cost per dose. Acquisition costs for each drug differed between the first year of treatment and subsequent years due to different dosing frequencies over the induction period (where the primary endpoints were assessed in phase III trials) compared with the maintenance period. Co-pay and co-insurance were not considered.

Costs for administering treatments to patients were also incurred based on dosing regimens for the applicable treatments. For tildrakizumab, all injections were administered at a clinic visit; all other subcutaneous treatments were administered at a clinic for the first visit and self-administered by the patients thereafter. Apremilast was administered orally and thus did not incur administration costs. The administration cost per treatment dose administered in a clinic setting was based on the physician fee schedule Current Procedural Terminology (CPT) code for subcutaneous injection [12].

Due to treatment interactions with the immune system, treated patients require laboratory testing at the initiation of treatment and periodically in each year of treatment to monitor for potential infection and liver function [6]. Therefore, annual costs of laboratory tests and patient monitoring were incurred for all treated patients. Annual laboratory resource use [i.e. latent tuberculosis (TB) screen, active TB screen, complete blood count, hepatitis B screen, liver function test, and renal function test] was based on estimated laboratory regimens [6]; costs per procedure or test were based on 2018 physician and laboratory fee schedules $[12,13]$. All treated patients were assumed to also require four annual clinic visits for disease and treatment monitoring [6]. Costs per clinic visit were based on the physician fee schedule CPT code for physician visits of intermediate complexity [12]. Annual frequencies of resource use were multiplied by the associated unit costs to derive annual resource costs (see Online Resource 1 for details). Costs of adverse events (AEs) were only included in scenario analyses, which is consistent with previous cost-effectiveness analyses [6].

All costs were adjusted to 2018 US dollars using the medical care component of the Consumer Price Index [14].

\subsection{Market Shares}

Current market shares before the introduction of tildrakizumab (scenario 1) were based on recent market estimates from the manufacturer of tildrakizumab assessed as of March 2019 (Table 1). In scenario 2, a 1\% annual uptake for tildrakizumab was assumed over 5 years based on manufacturer estimates; tildrakizumab market share was assumed to be taken proportionally from the other treatments so that the distribution of market shares across the other treatments remained the same as that in the current market.

Annual market shares were multiplied by the total number of patients treated each year to determine the number of patients receiving each treatment. Market shares were applied for the entire year, as patients did not switch or discontinue treatment during the year.

\subsection{Model Outcomes}

The budget impact analysis estimated costs based on the calculated number of patients treated, which remained constant over the 5-year period. Costs were calculated without discounting, as recommended by the International Society for Pharmacoeconomics and Outcome Research's Task Force on Good Research Practices for budget impact models [8]. For each treatment, costs were organized by drug costs, other costs, and overall costs, each year and cumulatively. Other costs included resource use costs for drug administration, laboratory procedures, and monitoring. Costs were reported as annual total costs for the health plan population, average annual costs per health plan member treated per year, PMPY, PMPM, and PTMPM. The incremental budget impact was calculated each year by comparing annual costs after the introduction of tildrakizumab (year 1 through year 5) with the annual costs for the current market without tildrakizumab.

Scenario analyses were performed to consider additional factors not included in the base case that may impact costs and to explore alternative assumptions used for the key inputs. Besides the direct drug-related costs, additional costs may be required to treat serious AEs associated with each treatment analyzed. An additional scenario including AEs due to treatment was considered. The incidence of hospitalizations due to severe infection, non-melanoma skin cancer (NMSC), and malignancies other than NMSC for biologic treatments and 
Table 1 Model inputs

\begin{tabular}{|c|c|c|c|c|c|c|}
\hline \multicolumn{7}{|l|}{ Plan population } \\
\hline \multicolumn{6}{|c|}{ Population characteristic } & Base case \\
\hline \multicolumn{6}{|c|}{ Number of members in health plan } & $1,000,000^{\mathrm{a}}$ \\
\hline \multicolumn{6}{|c|}{ Prevalence of psoriasis } & $3.2 \%^{\mathrm{b}}$ \\
\hline \multicolumn{6}{|c|}{ Proportion with plaque psoriasis } & $90.0 \%$ b \\
\hline \multicolumn{6}{|c|}{ Proportion with moderate-to-severe psoriasis } & $18.2 \%^{\mathrm{b}}$ \\
\hline \multicolumn{6}{|c|}{ Proportion treated with a biologic or apremilast } & $20.0 \%^{\mathrm{c}}$ \\
\hline \multicolumn{6}{|c|}{ New biologic or apremilast treatment users (require induction dosing) } & $35.6 \%$ d \\
\hline \multicolumn{7}{|c|}{ Annual drug costs } \\
\hline Treatment & Dose size $(\mathrm{mg})$ & $\begin{array}{l}\text { Cost per dose } \mathrm{e}^{\mathrm{e}} \\
(\text { WAC) (\$) }\end{array}$ & $\begin{array}{l}\text { Number of doses in } \\
\text { year } 1 \text { (per label) }\end{array}$ & $\begin{array}{l}\text { Average annual doses } \\
\text { in year } 2+\end{array}$ & $\begin{array}{l}\text { Total cost in } \\
\text { year } 1(\$)\end{array}$ & $\begin{array}{l}\text { Total cost in } \\
\text { year } 2+(\$)\end{array}$ \\
\hline Tildrakizumab & 100 & 13,256 & 5.0 & 4.3 & 66,279 & 57,000 \\
\hline Adalimumab & $40^{\mathrm{f}}$ & 2587 & 27.0 & 26.0 & 69,850 & 67,263 \\
\hline Apremilast & $30^{\mathrm{g}}$ & 57 & 729.0 & 730.0 & 41,286 & 41,342 \\
\hline Brodalumab & 210 & 1750 & 27.0 & 26.0 & 47,250 & 45,500 \\
\hline Etanercept & 50 & 1294 & 64.0 & 52.0 & 82,785 & 67,263 \\
\hline Guselkumab & 100 & 10,859 & 7.0 & 6.5 & 76,016 & 70,586 \\
\hline Ixekizumab & $80^{\mathrm{h}}$ & 5368 & 17.0 & 13.0 & 91,256 & 69,784 \\
\hline \multirow{2}{*}{$\begin{array}{l}\text { Secukinumab } \\
\text { Ustekinumab }\end{array}$} & 300 & 5179 & 16.0 & 13.0 & 82,863 & 67,326 \\
\hline & $45 / 90^{\mathrm{i}}$ & 16,503 & 5.0 & 4.3 & 82,517 & 70,965 \\
\hline \multicolumn{7}{|l|}{ Market shares } \\
\hline Treatment & Current $(\%)^{\mathrm{j}}$ & Year $1(\%)^{\mathrm{k}}$ & Year $2(\%)^{\mathrm{k}}$ & Year $3(\%)^{\mathrm{k}}$ & Year $4(\%)^{\mathrm{k}}$ & Year $5(\%)^{\mathrm{k}}$ \\
\hline Tildrakizumab & - & 1.0 & 2.0 & 3.0 & 4.0 & 5.0 \\
\hline Adalimumab & 26.7 & 26.5 & 26.2 & 25.9 & 25.7 & 25.4 \\
\hline \multirow{2}{*}{$\begin{array}{l}\text { Apremilast } \\
\text { Brodalumab }\end{array}$} & 16.9 & 16.7 & 16.6 & 16.4 & 16.2 & 16.1 \\
\hline & 0.6 & 0.6 & 0.6 & 0.6 & 0.6 & 0.6 \\
\hline Etanercept & 6.7 & 6.6 & 6.6 & 6.5 & 6.4 & 6.4 \\
\hline Guselkumab & 7.1 & 7.0 & 7.0 & 6.9 & 6.8 & 6.8 \\
\hline \multirow{2}{*}{$\begin{array}{l}\text { Ixekizumab } \\
\text { Secukinumab }\end{array}$} & 11.6 & 11.5 & 11.4 & 11.3 & 11.1 & 11.0 \\
\hline & 16.7 & 16.5 & 16.4 & 16.2 & 16.0 & 15.9 \\
\hline Ustekinumab & 13.6 & 13.5 & 13.3 & 13.2 & 13.1 & 12.9 \\
\hline
\end{tabular}

WAC wholesale acquisition cost

${ }^{a}$ A 1-million-member health plan is assumed

${ }^{b}$ Prevalence of psoriasis in the US is estimated at 3.2\% [2]. It is estimated that $90 \%$ of people with psoriasis have plaque psoriasis [1] and that $18 \%$ of those have moderate-to-severe plaque psoriasis [4]

${ }^{\mathrm{c}}$ It is assumed that $20 \%$ of patients with moderate-to-severe plaque psoriasis receive biologic treatment or apremilast, based on a study reporting that $17 \%$ of moderate-to-severe plaque psoriasis patients were currently treated, or treated in the last year, with biologics [10]

${ }^{\mathrm{d}}$ Thirty-six percent of biologic treatment users were assumed to be new to treatment each year $[6,7]$

${ }^{\mathrm{e}}$ Costs are based on May 2019 WAC prices [11] and recommended dosing from prescribing information; co-pay and co-insurance are not included. The incident population and patients switching biologic treatment incur drug acquisition costs for the treatment induction period (based on phase III clinical trial) and costs for doses administered during the maintenance period for the remainder of the year. The remainder of the treated population incurs drug acquisition costs based on the maintenance dosing regimens

$\mathrm{f} 80 \mathrm{mg}$ for the initial dose, followed by $40 \mathrm{mg}$ for all subsequent doses

${ }^{\mathrm{g}} 10 \mathrm{mg}$ for doses on days 1 and 2, and the morning of day 3, followed by $20 \mathrm{mg}$ for doses through the morning of day 5 , and then $30 \mathrm{mg}$ for all subsequent doses

${ }^{\mathrm{h}} 160 \mathrm{mg}$ for the initial dose, followed by $80 \mathrm{mg}$ for all subsequent doses

${ }^{\mathrm{i}}$ For patients weighing $\leq 100 \mathrm{~kg}, 45 \mathrm{mg}$ per dose; for patients weighing $>100 \mathrm{~kg}, 90 \mathrm{mg}$ per dose. It was assumed that $50 \%$ of patients received $45 \mathrm{mg}$ doses, while the remaining $50 \%$ received $90 \mathrm{mg}$ doses

${ }^{\mathrm{j}}$ Current market shares were based on March 2019 market share estimates from private communication with the manufacturer of tildrakizumab

${ }^{\mathrm{k}} \mathrm{A} 1 \%$ annual uptake for tildrakizumab was assumed over 5 years; the tildrakizumab market share was assumed to be taken proportionally from the other treatments so that the distribution of market shares across the other treatments remained the same as in the current market 
apremilast was included (see details in Online Resource 2). The incidence of AEs for adalimumab, etanercept, secukinumab, and ustekinumab was based on a technology appraisal for secukinumab [15], while the incidence for apremilast, guselkumab, and ixekizumab was based on prescribing information. The incidence for tildrakizumab was assumed to be the same as that for ustekinumab, as both are interleukin-23 antagonists. The costs of hospitalizations due to AEs were examined, with costs per hospitalization based on inpatient data for the relevant AEs from the 2015 Medicare inpatient charge data (see details in Online Resource 2) [16]. Another scenario analysis was conducted with a $2 \%$ annual uptake of tildrakizumab (versus $1 \%$ in the base case).

Sensitivity analysis was also performed to test the robustness of model outcomes and the uncertainty of model input data. A one-way sensitivity analysis was conducted by varying input values for psoriasis prevalence, percentage of patients requiring induction dosing, drug acquisition costs, and administration costs uniformly by $\pm 10 \%$, which was assumed to represent a reasonable range of uncertainty for the inputs varied. The effect of varying each input on the overall 5-year budget impact was measured, and the results were presented in a tornado diagram.

\section{Results}

\subsection{Base-Case Analysis}

For a health plan with 1 million members, it was estimated that 1048 people would be treated for moderate-to-severe plaque psoriasis each year, and 373 of those would be new to a biologic treatment or apremilast each year and require more frequent loading doses during the induction period (Table 2).

The total annual health plan costs associated with biologic treatments and apremilast were $\$ 70.67$ million for the current market without tildrakizumab. Total annual health plan costs after the introduction of tildrakizumab were $\$ 70.66$, $\$ 70.53$, $\$ 70.46, \$ 70.39$, and $\$ 70.33$ million in years 1 through 5, respectively. Annual health plan costs for tildrakizumab ranged from $\$ 700,000$ in year 1 to $\$ 3.19$ million in year 5. Average annual health plan costs per patient receiving treatment were $\$ 62,076$ for tildrakizumab, $\$ 68,710$ for adalimumab, $\$ 41,770$ for apremilast, $\$ 46,576$ for brodalumab, $\$ 73,357$ for etanercept, $\$ 72,981$ for guselkumab, $\$ 77,906$ for ixekizumab, $\$ 73,335$ for secukinumab, and $\$ 75,577$ for ustekinumab.

The cumulative health plan costs for tildrakizumab were $\$ 9.64$ million over 5 years, while overall costs across all treatments totaled approximately $\$ 352$ million $(\$ 67,227$ per treated patient per year). Of the total costs, $99.3 \%$ was attributed to drug acquisition costs, while the remaining $0.7 \%$ of costs were attributed to drug administration, laboratory procedures, and monitoring.
When comparing the market scenario with tildrakizumab and the market scenario without tildrakizumab, introducing tildrakizumab decreased the overall annual health plan cost by $\$ 5585, \$ 137,025, \$ 205,538, \$ 274,051$, and $\$ 342,563$ in years 1 through 5 , respectively, resulting in a cumulative reduction of $\$ 964,763$. The incremental budget impact projected total cost reductions between $0.01 \%$ and $0.48 \%$ in years $1-5$.

The incremental PMPY costs were $-\$ 0.01,-\$ 0.14,-\$ 0$. $21,-\$ 0.27$, and $-\$ 0.34$ in years $1-5$, respectively, resulting in a negligible impact on PMPM cost in year 1 , a reduction of $\$ 0.01$ in year 2, a reduction of $\$ 0.02$ in years $3-4$, and a reduction of $\$ 0.03$ in year 5 . The cumulative budget impact was - \$0.02 PMPM and - \$15.34 PTMPM.

\subsection{Scenario Analysis}

The scenario analysis results are presented in Fig. 1. Adding the costs of AEs to the analysis, the cumulative health plan costs were approximately $\$ 353$ million, while incremental annual health plan cost reductions were $\$ 6391, \$ 138,637$, $\$ 207,955, \$ 277,274$, and $\$ 346,592$ in years $1-5$, respectively. The cumulative incremental health plan cost reduction was $\$ 976,849$ when $\mathrm{AE}$ costs were included (versus $\$ 964,763$ in the base case), while cumulative PMPM budget impact changed negligibly from the base case.

Using a 2\% annual uptake for tildrakizumab, the incremental health plan cost reductions were $\$ 11,171, \$ 274,051$, $\$ 411,076, \$ 548,102$, and $\$ 685,127$ in years $1-5$, respectively. The cumulative 5-year health plan cost reductions from adding tildrakizumab to the formulary increased to $\$ 1,929,526$, while cumulative PMPM cost reductions increased to $\$ 0.03$ (versus $\$ 0.02$ in the base case).

\subsection{Sensitivity Analysis}

Figure 2 presents the impacts of key model parameters on the total annual costs over 5 years. The results were most sensitive to the drug acquisition costs per dose for tildrakizumab, followed by the acquisition costs per dose for adalimumab, ustekinumab, secukinumab, and apremilast. Variations in the acquisition costs per dose of brodalumab, proportion of patients requiring induction dosing, and drug administration costs had minimal impact.

\section{Discussion}

A budget impact analysis was conducted to estimate the impact of introducing and increasing the market share of tildrakizumab to a US health plan's budget over 5 years. To our knowledge, no prior analysis has assessed the potential budget impact of tildrakizumab for the treatment of moderate-to-severe plaque 
Table 2 Base-case results

\begin{tabular}{|c|c|c|c|c|c|c|c|}
\hline & Current & Year 1 & Year 2 & Year 3 & Year 4 & Year 5 & Cumulative $^{\mathrm{a}}$ \\
\hline Number receiving treatment & 1048 & 1048 & 1048 & 1048 & 1048 & 1048 & 5240 \\
\hline Number new to treatment & 373 & 373 & 373 & 373 & 373 & 373 & 1865 \\
\hline $\begin{array}{l}\text { Number receiving mainte- } \\
\text { nance treatment }\end{array}$ & 675 & 675 & 675 & 675 & 675 & 675 & 3375 \\
\hline Total annual costs ${ }^{\mathrm{b}}(\$)$ & $70,667,901$ & $70,662,315$ & $70,530,875$ & $70,462,363$ & $70,393,850$ & $70,325,337$ & $352,374,740$ \\
\hline Tildrakizumab & - & 701,094 & $1,276,333$ & $1,914,499$ & $2,552,665$ & $3,190,832$ & $9,635,422$ \\
\hline Drug costs & - & 694,817 & $1,264,344$ & $1,896,516$ & $2,528,688$ & $3,160,860$ & $9,545,226$ \\
\hline Other costs ${ }^{\mathrm{c}}$ & - & 6277 & 11,988 & 17,983 & 23,977 & 29,971 & 90,196 \\
\hline Adalimumab & $19,251,204$ & $19,058,692$ & $18,866,180$ & $18,673,668$ & $18,481,156$ & $18,288,644$ & $93,368,338$ \\
\hline Drug costs & $19,103,948$ & $18,912,908$ & $18,721,869$ & $18,530,830$ & $18,339,790$ & $18,148,751$ & $92,654,148$ \\
\hline Other costs ${ }^{\mathrm{c}}$ & 147,256 & 145,783 & 144,311 & 142,838 & 141,366 & 139,893 & 714,190 \\
\hline Apremilast & $7,407,648$ & $7,333,571$ & $7,259,495$ & $7,185,418$ & $7,111,342$ & $7,037,265$ & $35,927,091$ \\
\hline Drug costs & $7,328,215$ & $7,254,933$ & $7,181,651$ & $7,108,369$ & $7,035,087$ & $6,961,805$ & $35,541,845$ \\
\hline Other costs ${ }^{\mathrm{c}}$ & 79,432 & 78,638 & 77,844 & 77,049 & 76,255 & 75,461 & 385,246 \\
\hline Brodalumab & 293,252 & 290,320 & 287,387 & 284,455 & 281,522 & 278,590 & $1,422,274$ \\
\hline Drug costs & 290,400 & 287,496 & 284,592 & 281,688 & 278,784 & 275,880 & $1,408,442$ \\
\hline Other costs ${ }^{c}$ & 2852 & 2823 & 2795 & 2766 & 2738 & 2709 & 13,832 \\
\hline Etanercept & $5,157,532$ & $5,105,957$ & $5,054,381$ & $5,002,806$ & $4,951,231$ & $4,899,655$ & $25,014,030$ \\
\hline Drug costs & $5,117,628$ & $5,066,451$ & $5,015,275$ & $4,964,099$ & $4,912,923$ & $4,861,746$ & $24,820,494$ \\
\hline Other costs ${ }^{\mathrm{c}}$ & 39,904 & 39,505 & 39,106 & 38,707 & 38,308 & 37,909 & 193,536 \\
\hline Guselkumab & $5,437,449$ & $5,383,074$ & $5,328,700$ & $5,274,325$ & $5,219,951$ & $5,165,576$ & $26,371,626$ \\
\hline Drug costs & $5,403,080$ & $5,349,049$ & $5,295,018$ & $5,240,987$ & $5,186,957$ & $5,132,926$ & $26,204,937$ \\
\hline Other costs ${ }^{\mathrm{c}}$ & 34,369 & 34,025 & 33,682 & 33,338 & 32,994 & 32,651 & 166,690 \\
\hline Ixekizumab & $9,483,271$ & $9,388,438$ & $9,293,605$ & $9,198,773$ & $9,103,940$ & $9,009,107$ & $45,993,863$ \\
\hline Drug costs & $9,425,070$ & $9,330,819$ & $9,236,569$ & $9,142,318$ & $9,048,067$ & $8,953,817$ & $45,711,590$ \\
\hline Other costs ${ }^{\mathrm{c}}$ & 58,200 & 57,618 & 57,036 & 56,454 & 55,872 & 55,290 & 282,272 \\
\hline Secukinumab & $12,851,574$ & $12,723,058$ & $12,594,543$ & $12,466,027$ & $12,337,511$ & $12,208,995$ & $62,330,134$ \\
\hline Drug costs & $12,767,785$ & $12,640,108$ & $12,512,430$ & $12,384,752$ & $12,257,074$ & $12,129,396$ & $61,923,760$ \\
\hline Other costs ${ }^{c}$ & 83,789 & 82,951 & 82,113 & 81,275 & 80,437 & 79,599 & 406,375 \\
\hline Ustekinumab & $10,785,972$ & $10,678,112$ & $10,570,252$ & $10,462,392$ & $10,354,533$ & $10,246,673$ & $52,311,962$ \\
\hline Drug costs & $10,714,634$ & $10,607,487$ & $10,500,341$ & $10,393,195$ & $10,286,048$ & $10,178,902$ & $51,965,973$ \\
\hline Other costs ${ }^{\mathrm{c}}$ & 71,338 & 70,625 & 69,911 & 69,198 & 68,484 & 67,771 & 345,989 \\
\hline \multicolumn{8}{|c|}{ Incremental budget impact versus current market $(\$)$} \\
\hline Total costs & - & -5585 & $-137,025$ & $-205,538$ & $-274,051$ & $-342,563$ & $-964,763$ \\
\hline Total PMPY costs ${ }^{\mathrm{d}}$ & - & -0.01 & -0.14 & -0.21 & -0.27 & -0.34 & -0.19 \\
\hline Total PMPM costs ${ }^{\mathrm{d}}$ & - & 0.00 & -0.01 & -0.02 & -0.02 & -0.03 & -0.02 \\
\hline Total PTMPM costs ${ }^{\mathrm{d}}$ & - & -0.44 & -10.89 & -16.34 & -21.78 & -27.23 & -15.34 \\
\hline
\end{tabular}

$P M P M$ per member per month, $P M P Y$ per member per year, $P T M P M$ per treated member per month

${ }^{\mathrm{a}}$ Cumulative results are the sum of results from year 1 to year 5

${ }^{\mathrm{b}}$ Total annual costs include drug acquisition costs, administration costs, and monitoring (laboratory tests and clinic visits) costs

${ }^{\mathrm{c}}$ Other costs included treatment administration, laboratory, and monitoring

${ }^{\mathrm{d}}$ PMPY, PMPM, and PTMPM costs are based on a 1-million-member plan. 'Treated members' includes all patients with moderate-to-severe plaque psoriasis treated with biologic treatments or apremilast. The costs listed in the 'Cumulative' column are the average PMPY, PMPM, and PTMPM costs over the 5-year time horizon

psoriasis in the US. The results of the analysis showed that, for the assumed market uptake and market shares of other treatments, the introduction of tildrakizumab had a minimal impact on a health plan's costs. Overall health plan costs were almost completely attributed to acquisition costs for biologic treatments and apremilast. Compared with a market scenario without tildrakizumab, the total health plan costs would decrease from the first year forward after introducing tildrakizumab to the market for the treatment of moderate-to-severe plaque psoriasis. 
Fig. 1 Scenario analysis results. Note: annual and cumulative incremental budget impacts are shown above. The 'AEs included' scenario includes adverse event-related hospitalizations for serious infection, NMSC, and malignancies other than NMSC. The 'higher tildrakizumab uptake' scenario considers $2 \%$ annual market share uptake for tildrakizumab (versus $1 \%$ annual uptake in the base case). $A E$ adverse events, NMSC non-melanoma skin cancer

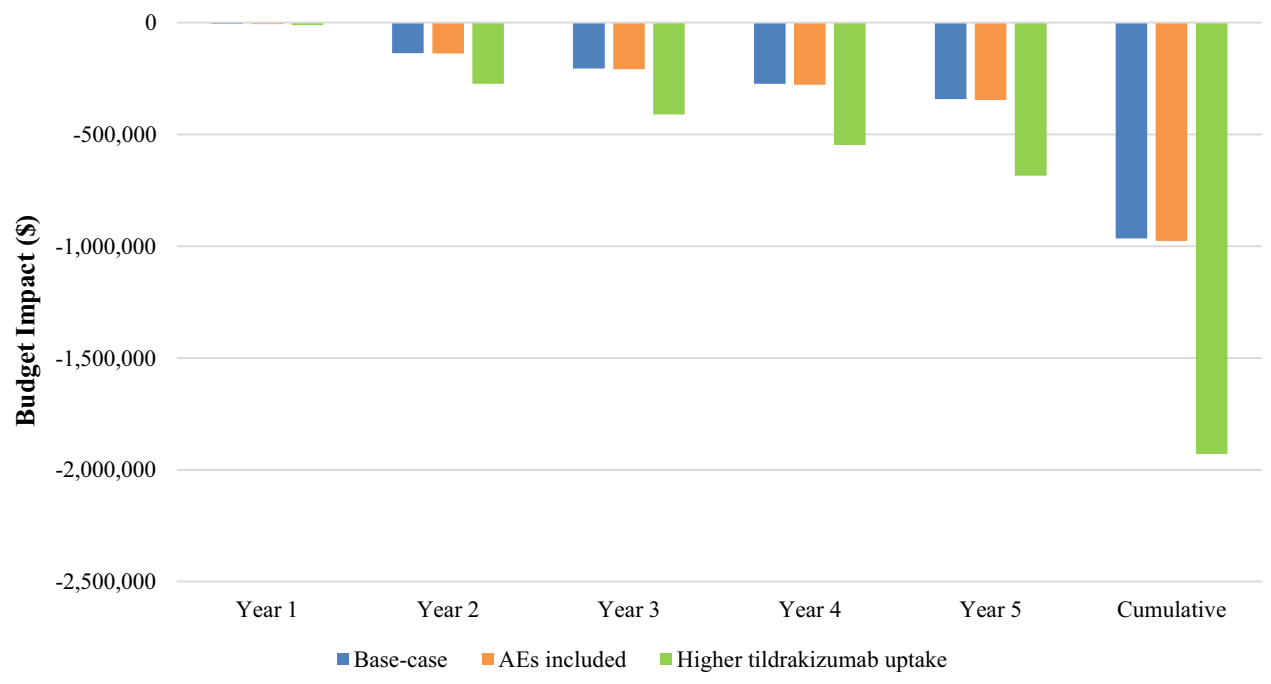

Tildrakizumab acquisition cost per dose $(\$ 11,930-\$ 14,581)$

Adalimumab acquisition cost per dose $(\$ 2,328-\$ 2,846)$

Secukinumab acquisition cost per dose $(\$ 4,661-\$ 5,697)$

Ustekinumab acquisition cost per dose $(\$ 14,853-\$ 18,154)$

氙

Ixekizumab acquisition cost per dose $(\$ 4,831-\$ 5,905)$

Apremilast acquisition cost per dose (\$51-\$62)

Prevalence of psoriasis $(2.9 \%-3.5 \%)$

Guselkumab acquisition cost per dose $(\$ 9,774-\$ 11,945)$

Etanercept acquisition cost per dose $(\$ 1,164-\$ 1,423)$

Brodalumab acquisition cost per dose $(\$ 1,575-\$ 1,925)$

Subcutaneous administration cost per dose (\$24-\$29)

$\%$ of treatment users requiring induction dosing $(32.0 \%-39.2 \%)$

口Upper bound $\quad$ Lower bound

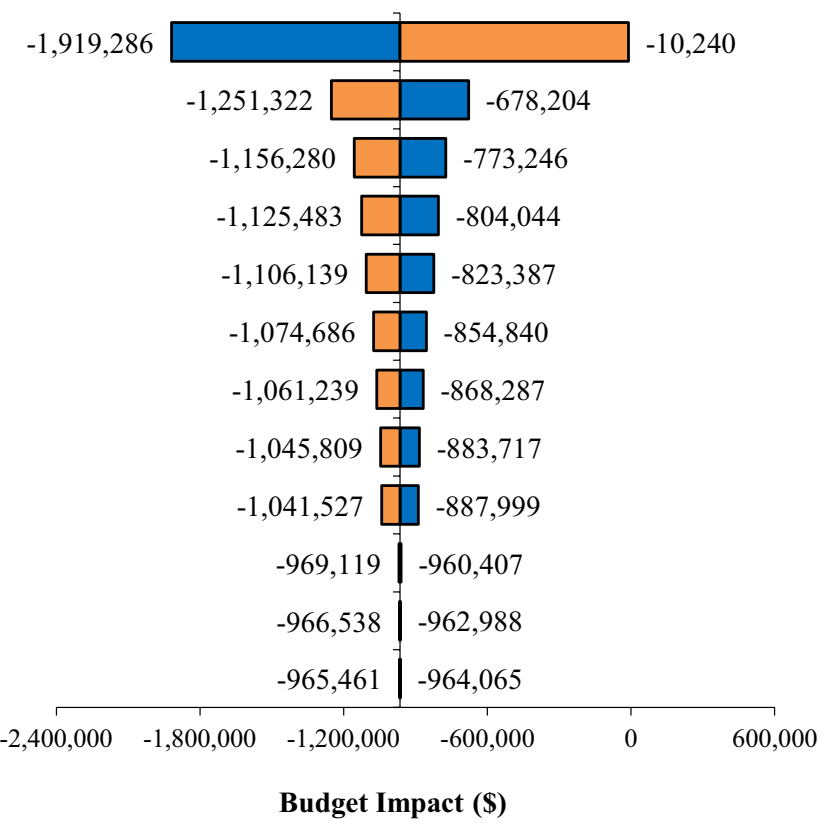

Fig. 2 One-way sensitivity analysis: impact of changes of input values on the cumulative budget impact over 5 years. Note: All inputs were varied uniformly by $\pm 10 \%$ of the base-case values

Incremental PMPM health plan costs decreased cumulatively by $\$ 0.02$ over 5 years.

The results were largely driven by differences in drug acquisition costs. The smaller decrease in total cost in the first year of tildrakizumab introduction, compared with subsequent years, was attributable to higher drug acquisition costs due to more frequent dosing during the induction period for tildrakizumab patients, who were all assumed to be new to treatment in its first year of introduction. After the first year, patients continuing treatment with tildrakizumab only needed to receive maintenance dosing and thus total costs decreased more significantly versus the current treatment market as the uptake of tildrakizumab continued. Tildrakizumab annual drug acquisition cost per patient in maintenance treatment $(\$ 57,000)$ compared favorably with the biologic treatments with the largest market shares $\$ 67,263$ for adalimumab and $\$ 67,326$ for secukinumab). This contributed to the decreases in total health care costs and PMPM costs during the subsequent years of tildrakizumab introduction.

The impact of AE costs was not considered in the basecase analysis but was shown to have minimal effect on budget impact results in the scenario analysis. Scenario analysis also demonstrated that higher uptake of tildrakizumab may further reduce total health plan costs for plaque psoriasis treatment, especially when market share is taken from drugs with more expensive annual acquisition costs 
for maintenance treatment, such as adalimumab, etanercept, guselkumab, ixekizumab, secukinumab, and ustekinumab.

While previous studies have examined the budget impact for various psoriasis treatments, few analyses have been conducted in the US, and no studies have included tildrakizumab among the treatment options. A recent study modeled the potential budget impact of brodalumab in the US [17]. Across all modeled treatments, overall annual health plan costs for the treatment of moderate-to-severe plaque psoriasis were slightly higher per treated patient per year in the current study (approximately $\$ 67,000)$ than in the study by Feldman and colleagues (approximately \$59,000) [17] as more recent and higher drug acquisition data were used in the current study. In addition, Feldman and colleagues considered patient co-pays and drug discounts for drug acquisition costs, which reduced the overall costs borne by health care payers; to simplify the analyses, these factors were not considered in the current study.

\subsection{Limitations}

Population-related parameters for the model were based on national data and may not reflect the population characteristics of individual health plans. The prevalence of moderateto-severe plaque psoriasis was assumed to remain constant over the modelled time horizon. The World Health Organization (WHO) 2016 Global Report on Psoriasis noted that US data indicated an increase in prevalence from $2.2 \%$ in 2004 to $3.2 \%$ in 2010 [18]. However, the WHO noted that differences in methodologies for prevalence studies included in the report do not support a conclusive statement that psoriasis prevalence is increasing over time. In addition, sensitivity analysis demonstrated that changes in prevalence rates and other population characteristics affect the scale of the overall health plan costs and not the magnitude of the budget impact from introducing tildrakizumab.

As drug acquisition costs were based on recent WACs, costs for individual health plans will differ based on co-pay, co-insurance, and use of other treatments not included in this analysis, such as biosimilars. Manufacturer discounts or rebates may also affect drug acquisition costs. However, actual discounts or rebate amounts are often proprietary information and can be variable across markets and were therefore not considered.

The market shares for treatments other than tildrakizumab included in the model remained constant over the 5-year time horizon, whereas market shares may change over time in the real world and market landscapes for actual US health plans will likely continue to change over time as new treatments enter the market. Uptake of biosimilars was not considered, as none of the treatments in this analysis have biosimilars that are currently marketed in the US. Therefore, market uptake and pricing data were not available. The exclusion of biosimilars is in line with the recent budget impact analysis of plaque psoriasis treatment by Feldman et al. [17], which also excluded biosimilars due to uncertainty in real-world substitution of brand name drugs.

AE costs were only considered in scenario analysis due to similar AE profiles between treatments and low incidence rates for serious AEs. Scenario analysis demonstrated that the inclusion of AEs had a negligible impact on overall health plan costs and incremental health plan costs associated with the introduction of tildrakizumab.

This budget impact analysis did not consider treatment responses and assumed $100 \%$ adherence to therapy within each year of the modeled time horizon. Previous economic analyses did not consider cost implications of differences in treatment response [6, 17], but such differences could impact costs if more effective treatment is associated with decreased health care resource use. A recent study estimated an overall adherence rate of $76 \%$ for biologics used to treat psoriasis and did not find statistically significant differences in adherence between the treatments observed [19], but did not include all treatments modelled in this analysis. Differences in adherence across treatments could impact health plan costs if the frequency of treatment administration is impacted or if additional medical visits are required for nonadherent patients. Although the number of patients discontinuing treatment was not captured, annual market shares were assumed to capture patient movements across treatments due to lack of treatment response.

\section{Conclusions}

The introduction of tildrakizumab, with a $1 \%$ annual uptake over 5 years, is expected to have a small financial impact on health plan costs and may reduce the cost of treating patients with moderate-to-severe plaque psoriasis for a US health plan. Results were consistent across scenarios tested; however, future uptake of biosimilars may impact overall health plan costs for plaque psoriasis treatment.

Author contributions JC, XJ, YZ, and THB were responsible for conception and design of the research. Economic modeling was carried out by $\mathrm{JC}, \mathrm{XJ}$, and THB, and acquisition of data was carried out by JC, XJ, and YZ. JC, XJ, YZ, and THB were responsible for development of the draft manuscript, and all authors were responsible for critical revision of the manuscript for important intellectual content.

Data availability statement All data generated or analyzed during this study are included in this published article (and its supplementary information files).

\section{Compliance with Ethical Standards}

Funding Sun Pharmaceutical Industries, Inc. provided funding for all research contributing to this manuscript, and was also involved in the design and conduct of the study; collection, management, and analysis of the data; and preparation, review, and approval of the manuscript. 
Financial/other interests YZ, AM, and SL were employees of Sun Pharmaceutical Industries, Inc. at the time of this analysis. XJ, JC, and THB are employees of RTI-Health Solutions, all of whom were contracted by Sun Pharmaceutical Industries, Inc. to support the study.

Previous publication of research Carrico J, Zhao Y, Jia X, Brodtkorb TH, Mendelsohn A, Lowry S. Budget Impact of Introducing Tildrakizumab for the Treatment of Moderate-to-Severe Plaque Psoriasis in the United States. Poster presented at the Academy of Managed Care Pharmacy (AMCP) Nexus; 22-25 October 2018; Orlando, FL.

Open Access This article is licensed under a Creative Commons Attribution-NonCommercial 4.0 International License, which permits any non-commercial use, sharing, adaptation, distribution and reproduction in any medium or format, as long as you give appropriate credit to the original author(s) and the source, provide a link to the Creative Commons licence, and indicate if changes were made. The images or other third party material in this article are included in the article's Creative Commons licence, unless indicated otherwise in a credit line to the material. If material is not included in the article's Creative Commons licence and your intended use is not permitted by statutory regulation or exceeds the permitted use, you will need to obtain permission directly from the copyright holder.To view a copy of this licence, visit http://creativecommons.org/licenses/by-nc/4.0/.

\section{References}

1. Boehncke WH, Schon MP. Psoriasis. Lancet. 2015;386(9997):983-94.

2. Rachakonda TD, Schupp CW, Armstrong AW. Psoriasis prevalence among adults in the United States. J Am Acad Dermatol. 2014;70(3):512-6.

3. Brezinski EA, Dhillon JS, Armstrong AW. Economic burden of psoriasis in the United States: a systematic review. JAMA Dermatol. 2015;151(6):651-8.

4. Helmick CG, Lee-Han H, Hirsch SC, et al. Prevalence of psoriasis among adults in the US: 2003-2006 and 2009-2010 National Health and Nutrition Examination Surveys. Am J Prev Med. 2014;47(1):37-45.

5. Mrowietz U, Kragballe K, Reich K, et al. Definition of treatment goals for moderate to severe psoriasis: a European consensus. Arch Dermatol Res. 2011;303(1):1-10.

6. Hendrix N, Ollendorf DA, Chapman RH, et al. Cost-effectiveness of targeted pharmacotherapy for moderate to severe plaque psoriasis. J Manag Care Spec Pharm. 2018;24(12):1210-7.
7. Feldman SR, Zhao Y, Navaratnam P, et al. Patterns of medication utilization and costs associated with the use of etanercept, adalimumab, and ustekinumab in the management of moderateto-severe psoriasis. J Manag Care Spec Pharm. 2015;21(3):201-9.

8. Sullivan SD, Mauskopf JA, Augustovski F, et al. Budget impact analysis-principles of good practice: report of the ISPOR 2012 Budget Impact Analysis Good Practice II Task Force. Value Health. 2014;17(1):5-14.

9. Reich K, Papp KA, Blauvelt A, et al. Tildrakizumab versus placebo or etanercept for chronic plaque psoriasis (reSURFACE 1 and reSURFACE 2): results from two randomised controlled, phase 3 trials. Lancet. 2017;390(10091):276-88.

10. Armstrong AW, Koning JW, Rowse S, Tan H, Mamolo C, Kaur M. Under-treatment of patients with moderate to severe psoriasis in the United States: analysis of medication usage with health plan data. Dermatol Ther. 2017;7(1):97-109.

11. Micromedex. Red book online. Greenwood Village: Truven Health Analytics. http://www.micromedexsolutions.com/. Accessed 14 May 2019.

12. Centers for Medicare \& Medicaid Services. Physician fee schedule. 2017. https://www.cms.gov/apps/physician-fee-schedule/. Accessed 25 Sep 2017.

13. Centers for Medicare \& Medicaid Services. Clinical diagnostic laboratory fee schedule. 2017. https://www.cms.gov/Medicare/ Medicare-Fee-for-Service-Payment/ClinicalLabFeeSched/index .html. Accessed 25 Sep 2017.

14. United States Bureau of Labor Statistics. Bureau of labor statistics consumer price index-all urban consumers. 2018. https://www. bls.gov/cpi/data.htm. Accessed 14 Feb 2018.

15. National Institute for Health and Care Excellence (NICE). Single technology appraisal. Secukinumab for treating moderate to severe plaque psoriasis [TA350]. 2015. https://www.nice.org.uk/ guidance/ta350. Accessed 27 Jul 2017.

16. Centers for Medicare \& Medicaid Services. National and state summaries of inpatient charge data, FY2015. 2017. https://www. cms.gov/Research-Statistics-Data-and-Systems/Statistics-Trend s-and-Reports/Medicare-Provider-Charge-Data/Inpatient2015. html. Accessed 3 Oct 2017.

17. Feldman SR, Wu JJ, Rastogi S, et al. The budget impact of brodalumab for the treatment of moderate-to-severe plaque psoriasis on US commercial health plans. J Med Econ. 2018;21(5):537-41.

18. World Health Organization. Global report on psoriasis. 2016. https ://apps.who.int/iris/handle/10665/204417. Accessed 24 Jun 2019.

19. Mocanu M, Toader MP, Rezus E, Taranu T. Aspects concerning patient adherence to anti-TNF $\alpha$ therapy in psoriasis: a decade of clinical experience. Exp Ther Med. 2019;18(6):4987-92.

\section{Affiliations}

\section{Justin Carrico ${ }^{1} \cdot$ Yang Zhao $^{2} \cdot$ Xiaoying Jia $^{3} \cdot$ Thor-Henrik Brodtkorb $^{4} \cdot$ Alan Mendelsohn $^{5} \cdot$ Simon Lowry $^{6}$}

\author{
Justin Carrico \\ jcarrico@ rti.org \\ Xiaoying Jia \\ louisejia@rti.org \\ Thor-Henrik Brodtkorb \\ tbrodtkorb@rti.org
}

Alan Mendelsohn

Alan.Mendelsohn@sunpharma.com

Simon Lowry

silowry@yahoo.com
1 RTI Health Solutions, Research Triangle Park, NC, USA

2 Kyowa Kirin Pharmaceutical Development, Inc., 212 Carnegie Center Dr \#400, Princeton, NJ 08540, USA

3 RTI Health Solutions, Manchester, UK

4 RTI Health Solutions, Ljungskile, Sweden

5 Sun Pharmaceutical Industries, Inc., Princeton, NJ, USA

6 Roivant Sciences Ltd, New York, NY, USA 